Thorax (1950), 5, 343.

\title{
FIFTY-SEVEN CASES OF DIAPHRAGMATIC HERNIA AND EVENTRATION
}

\author{
BY \\ C. J. EVANS AND J. A. SIMPSON \\ From the Regional Thoracic Surgery Centre, Shotley Bridge Hospital, \\ Newcastle-upon-Tyne
}

(RECEIVED FOR PUBLICATION JULY 8, 1950)

During the last 20 years great advances have been made in the recognition of diaphragmatic hernia, and many series of cases reported by numerous authors have helped to place the diagnosis and treatment on a sound surgical basis (Dunhill, 1935 ; Barrett and Wheaton, 1934 ; Donovan, 1945 ; Morton, 1939 ; Harrington, 1940 ; Gross, 1946). More recently progress in thoracic surgery and anaesthesia has given a more direct approach to the lesion itself and has placed the treatment of diaphragmatic hernia on a basis comparable with the treatment of other types of herniation of the abdominal organs (Sweet, 1948 ; Truesdale, 1945 ; Allison, 1946).

Since 1937, 46 cases of diaphragmatic hernia have been admitted to this unit. These are reviewed, and three additional cases, treated by Mr. G. A. Mason at the Nottingham General Hospital, have been included in the series. They are classified as (1) traumatic hernia (a) due to non-penetrating injuries; $(b)$ due to penetrating injuries: (c) post-operative; and (2) non-traumatic herniae: (a) oesophageal hiatus hernia with normal oesophagus; $(b)$ oesophageal hiatus hernia with pathological changes in the oesophagus; and $(c)$ hernia through defects in the muscular and tendinous portions of the diaphragm.

The subdivision of oesophageal hiatus hernia into those with and those without pathological changes in the oesophagus is necessary in view of the different problems of diagnosis and treatment involved.

No attempt has been made to classify the cases into congenital and acquired types ; accurate differentiation is not always possible and is of academic interest only.

Eight cases of eventration of the diaphragm seen over the same period are also reviewed.

\section{Traumatic Hernia}

Due to Non-Penetrating Injuries (7 Cases).-Severe crushing injuries to the abdomen accounted for five cases, and four of these also sustained a fractured pelvis. In none did coincident rupture of any abdominal viscera occur, although in one patient a laparotomy had been performed elsewhere with this suspected diagnosis. Only one sustained bruising of the chest wall and none had fractured ribs, which suggests that the mechanism of injury to the diaphragm is in the nature of an explosive rupture due to sudden increase in intra-abdominal pressure.

Case 1 (No. 5307). - A woman aged 39 was involved in a car accident and thrown violently forwards against the driver's seat. She was seven and a half months pregnant 


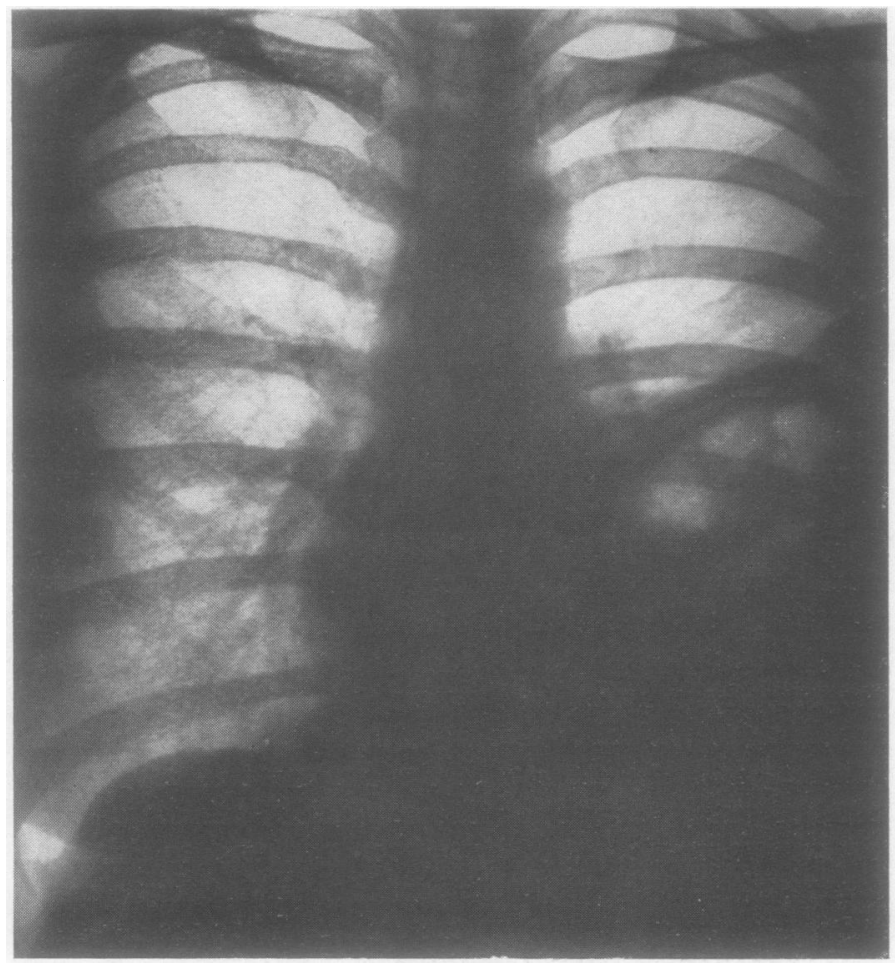

FIG. 1.-Kadiograph of the chest (Case 1) taken one month after a crushing injury to the abdomen and showing a left diaphragmatic hernia.

The diaphragm may be ruptured by less violent trauma. Two patients whose symptoms followed immediately after a fall downstairs were discovered later to have a diaphragmatic hernia. The left diaphragm was ruptured in six cases, and the right in one, as shown diagrammatically in Fig. X.

The clinical picture depends on the nature of the viscera entering the chest, the rapidity with which they enter and the disturbance or otherwise of their function, and also on the displacement of the lungs and mediastinum which is produced. Cases may be seen as emergencies soon after injury with symptoms referable to the cardiovascular, respiratory, or alimentary systems, or they may be seen months or years later with less urgent symptoms referable mainly to the alimentary tract.

Respiratory distress, cyanosis, and tachycardia (Cases 2 and 3) occurred immediately after injury in two cases. Harrington (1945) and many other authors have emphasized that in such circumstances operative reduction should be performed without delay as this alone will relieve their symptoms.

Case 2 (No. 3920).-A girl aged 8 was knocked down by a lorry. On examination a few hours afterwards she was cyanosed, had rapid, grunting respirations, and an irritating cough. There was bruising of the abdomen and left hip and diffuse abdominal tenderness. Radiographs of the pelvis showed wide separation of the pubic symphysis with fracture of the ischial ramus. Chest films were suggestive of herniation of the 


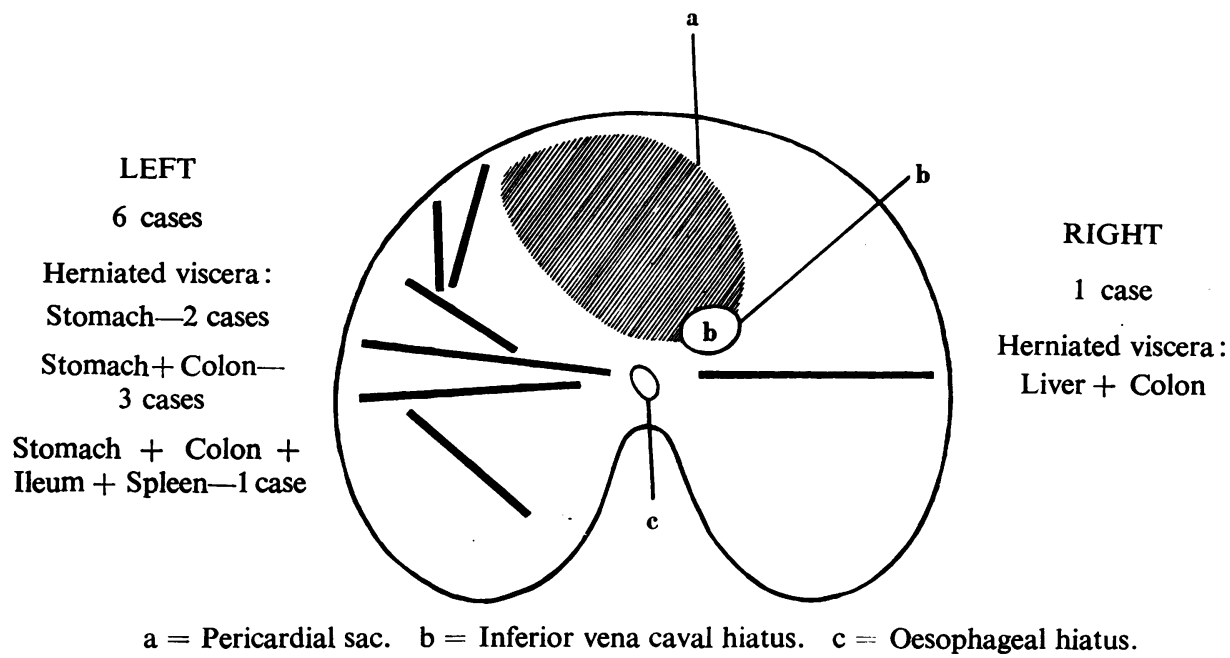

FIG.X.-Diagram of diaphragm as seen from above, showing sites of rupture due to non-penetrating injuries.

liver into the chest (Fig. 2), and a right thoracotomy was performed. Loops of colon and most of the liver were found herniated through a large tear in the dome of the right diaphragm. A tear was also found in the pericardial sac. The viscera were replaced and the hernia repaired. There was an immediate improvement in the child's colour and general condition and she made a good recovery.

Dugan and Samson in 1948 reported two cases of strangulation of the stomach in a traumatic diaphragmatic hernia and collected another four examples from the literature. Orr also reported a case in 1946.

Immediate obstruction of the stomach occurred in one of our patients (Case 3), and reduction proved impossible by the abdominal route.

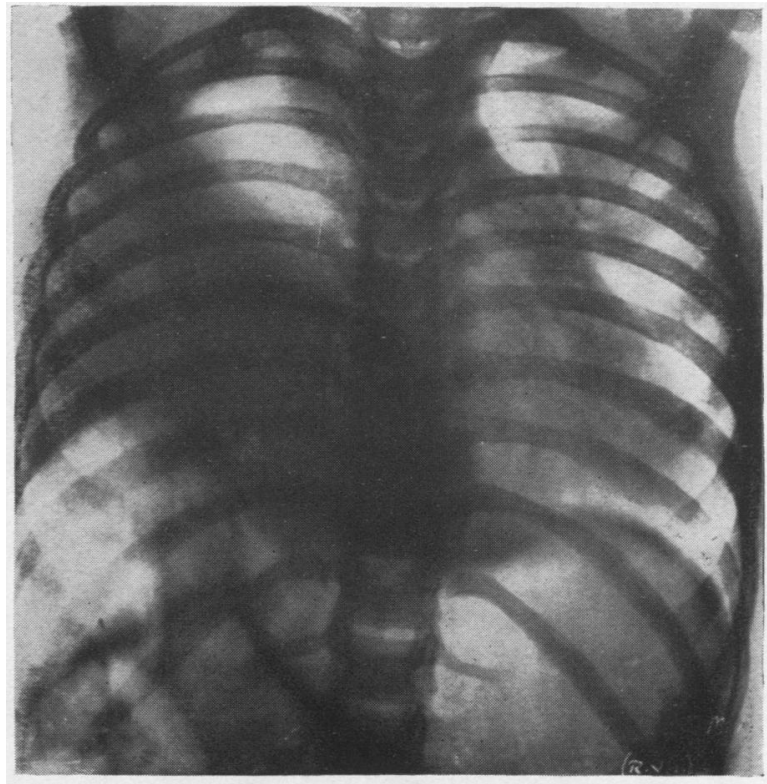

FIG. 2.-Radiograph (Case 2) showing herniation of the liver and of the hepatic flexure of the colon into the right hemithorax. There is a dense homogeneous opacity in the middle and lower zones of the right hemithorax with translucency of the right upper quadrant of the abdomen and an abnormally high position of the gas shadow in the right colon. The mediastinum is displaced to the left. 
Case 3 (No. 3876).-A boy of 16 was run over by a car and sustained a crush injury to the abdomen and a fractured pelvis. A ruptured viscus was suspected. At laparotomy a retroperitoneal haematoma and an irreducible diaphragmatic hernia were found and the abdomen was closed. Three days later he was admitted to the thoracic unit. He was cyanosed and had a weak, rapid pulse. He was vomiting brownish fluid, and his abdomen was slightly distended. Radiographs showed the left hemithorax occupied by distended stomach with displacement of the mediastinum to the right (Fig. 3).

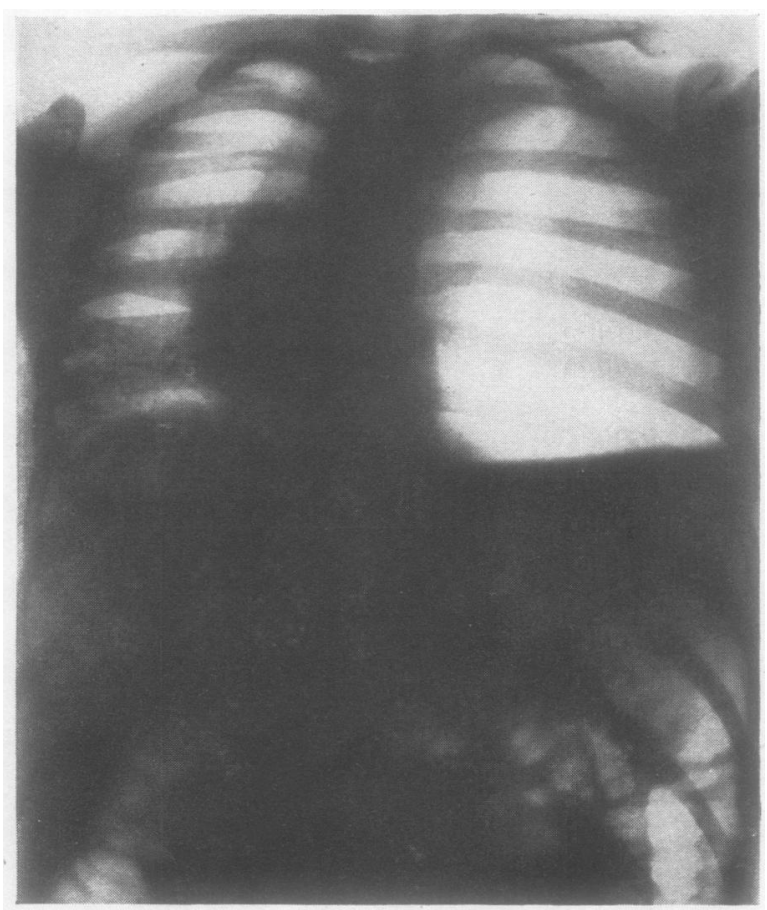

FIG. 3.-Radiograph (Case 3) showing the left hemithorax occupied by a grossly distended stomach with collapse of the left lung and displacement of the mediastinum to the right.

Obstruction at the oesophagogastric junction prevented passage of a stomach tube. A left thoracotomy was performed and a grossly distended stomach presented. Reduction was not possible until the stomach had been deflated with an aspirating needle. The tear in the diaphragm extended from near the oesophageal hiatus to the lateral chest wall and was sutured in two layers. His colour was greatly improved at the end of the operation. He died 36 hours later from paralytic ileus.

The possibility of diaphragmatic hernia should be borne in mind after any crushing injury of the abdomen, and barium radiological examination carried out even in the absence of symptoms. Four patients were first seen from one to seven years after injury. All had symptoms which were increasing in severity. In two these dated from the time of injury, but in the other two the symptoms had been delayed for intervals of six months and two years after the injury. All complained of pain, situated either in the epigastrium or the lower part of the left chest ; it was intermittent and usually brought on or aggravated by food. In one patient attacks of severe pain were precipitated by the lifting of heavy weights. Acute obstructive attacks with vomiting and eructations occurred in one who was later found to have herniation of a large portion of the stomach through a relatively narrow hernial orifice. Another patient had noticed rumblings in his chest. All admitted to some degree of dyspnoea on exertion and one had complained of cough and muco-purulent sputum since the injury. In these four patients straight anteroposterior and lateral radiographs were sufficient to make a diagnosis, and additional confirmation was obtained by barium examination.

Operative repair by the transthoracic route was undertaken in all. As emphasized by Hughes, Kay, Meade, Hudson, and Johnson (1948) this approach offers a 
better exposure than is possible from the abdomen, permits safe division of adhesions, and facilitates closure of the defect.

There were two deaths and no recurrences in this group. One (Case 3) died of paralytic ileus. The other developed a post-operative staphylococcal empyema which was drained. Four months later, when the empyema had healed, he died suddenly and necropsy revealed thrombosis of the abdominal aorta. The diaphragm was found to be soundly healed.

Due to Penetrating Injuries (4 Cases).-One patient was seen a few hours after injury, with a penetrating wound of the lower part of his left chest through which omentum presented. A left thoracotomy was performed. The omentum had entered the chest through a wound, 1 in. in diameter, in the dome of the left diaphragm. This wound was enlarged to permit suture of a laceration of the left lobe of the liver. The diaphragm was repaired in two layers and he made a good recovery.

Two patients were operated on three months after sustaining penetrating gunshot wounds. In one the hernia was diagnosed pre-operatively; in the other it was an accidental finding during an operation for removal of a foreign body embedded in the lower lobe of the left lung. In each a portion of the stomach had herniated through a small defect in the dome of the left diaphragm. Both patients made an uneventful recovery.

The fourth patient had a recurrent hernia in which the repair of a large defect proved impossible by simple suture.

Case 4 (No. 5707).-A man, aged 67, sustained a bullet wound of the left chest in 1915 and had a fascial repair of a diaphragmatic hernia in 1921. He was well until 1949 when he began to have attacks of colicky epigastric pain. On examination there was scarring and an underlying deficiency of the thoracic cage in the region of the sixth and seventh ribs antero-laterally. This area bulged on abdominal pressure and exhibited paradoxical movements on respiration. Radiography revealed a large diaphragmatic hernia containing the whole of the stomach and splenic flexure in the left pleural cavity. At operation the herniated viscera were replaced after division of dense adhesions. A large elliptical defect in the diaphragm was found to extend from the oesophageal orifice antero-laterally to the chest wall. The defect was too wide to close by simple suture. Segments of the eighth, ninth, and tenth ribs were resected in order to mobilize the attachments of the diaphragm and enable the edges of the defect to be approximated. The intercostal bundles were used to reinforce the suture line. The patient died six hours later without recovering consciousness. Permission for necropsy was not granted.

Post-Operative Hernia.- There was one case of herniation following cardioomentopexy, in which strangulation of the large intestine occurred.

Case 5 (No. 234).-A man, aged 52, in May, 1937, had a transpleural cardioomentopexy for angina pectoris, and following this operation the symptoms were relieved. He was well until December, 1937, when he was readmitted with a history of severe abdominal pain, vomiting, and absolute constipation of three days' duration. On examination the abdomen was distended, and auscultation over the praecordium revealed loud and persistent borborygmi. A left thoracotomy was performed and the pleural cavity was found to contain gas under pressure, blood-stained fluid, and about one foot of transverse colon which had herniated through the diaphragm with the omental graft. The bowel was grossly distended but appeared viable. The hernial orifice was enlarged 
and the bowel returned to the abdomen. The omental graft looked healthy and its vessels were felt to pulsate; the opening in the diaphragm was sutured around it. A caecostomy was performed. The patient died five days later from paralytic ileus and bronchopneumonia.

\section{Non-Traumatic Hernia}

Oesophageal Hiatus Hernia with Normal Oesophagus (19 Cases).-Subdivision into para-oesophageal and sliding gastro-oesophageal types is of no practical importance in our opinion. In all cases the oesophagus was normal and permitted replacement of the oesophago-gastric junction below the diaphragm.

Seventeen of the patients were women and two men. Four were aged between 20 and 40, and 14 between 47 and 75 years. There was one child aged 4.

Case 6 (No. 5363).-This was a boy aged 4 years with a history of feeding difficulties and bouts of vomiting since birth. Three months before admission he was reported to have brought up altered blood during an attack of vomiting lasting several days. On admission he was in good general condition and well developed for his age. Straight radiographs showed a hiatus hernia and this was confirmed by barium examinations. At operation the hernial sac contained stomach and transverse colon, and the oesophageal hiatus was found to be enlarged to 2 in in diameter. Reduction and repair were effected by the technique described below. He made a good recovery and has remained free of symptoms.

In this group the duration of symptoms varied from months to years, more often the latter. All patients complained of pain or discomfort, usually situated in the epigastrium or lower chest on the left side. Other sites were the praecordium and interscapular region. In one patient pain was referred to the left shoulder. The pain varied in severity and most often occurred in attacks immediately or soon after food. In another patient food relieved the pain, but most were more comfortable on small meals taken at frequent intervals. Some loss of weight was usual. Vomiting and eructations were common features and often relieved pain. No patient in this group complained of difficulty in swallowing. Aggravation of symptoms on recumbency was noted in two, and one of these complained of attacks of vomiting soon after going to bed, but another said that recumbency relieved her pain. One patient had noticed a splashing sensation in her chest.

In all cases of abdominal symptoms of obscure origin the possibility of hiatus hernia should be considered (Olsen and Harrington, 1948). In two of our patients the symptoms had been mistaken for gall-bladder dyspepsia and one had had a cholecystectomy performed without relief. In a third the pain had been thought to be due to coronary artery disease.

In a woman, aged 69, an ulcer was demonstrated pre-operatively on the lesser curvature, where the stomach was constricted by the hiatal ring, and its presence was confirmed at operation. Repair of the hernia was followed by relief of symptoms and radiological regression of the ulcer. Another woman, aged 67, who gave a characteristic history of hiatus hernia, with in addition repeated recent small haematemeses, was found at operation to have an active chronic duodenal ulcer. The hernia was repaired and vagotomy performed in March, 1950. She has obtained complete symptomatic relief to date. Another patient had had a large haematemesis, but no cause for this could be found at operation. 
In three patients acute obstructive attacks had occurred. In two vomiting was severe and the vomitus contained altered blood; relief was obtained by the passage of a stomach tube. In the third (Case 7) closed loop obstruction of the stomach occurred.

Case 7 (No. 5295).-A woman, aged 56, gave a four months' history of attacks of epigastric pain, vomiting, and abdominal distension. Three months before admission a laparotomy had been performed for a suspected perforated ulcer. A diaphragmatic hernia, but no perforation, was found and the abdomen was closed. One month before admission she was reported to have had a small haematemesis. She was admitted as an emergency with abdominal pain, vomiting, and gross abdominal distension. Radiographs showed enormous distension of the body of the stomach (Fig. 4). A stomach tube was passed and a large quantity of gas and fluid withdrawn. After preparation with intravenous fluids and continuous gastric suction a left thoracotomy was performed. The sac of the hiatal hernia, lying posterior and to the right of the lower end of the oesophagus, was found to contain the pyloric antrum and the pylorus. The contents were reduced after division of a few adhesions to the neck of the sac. A repair was performed according to the technique to be described.

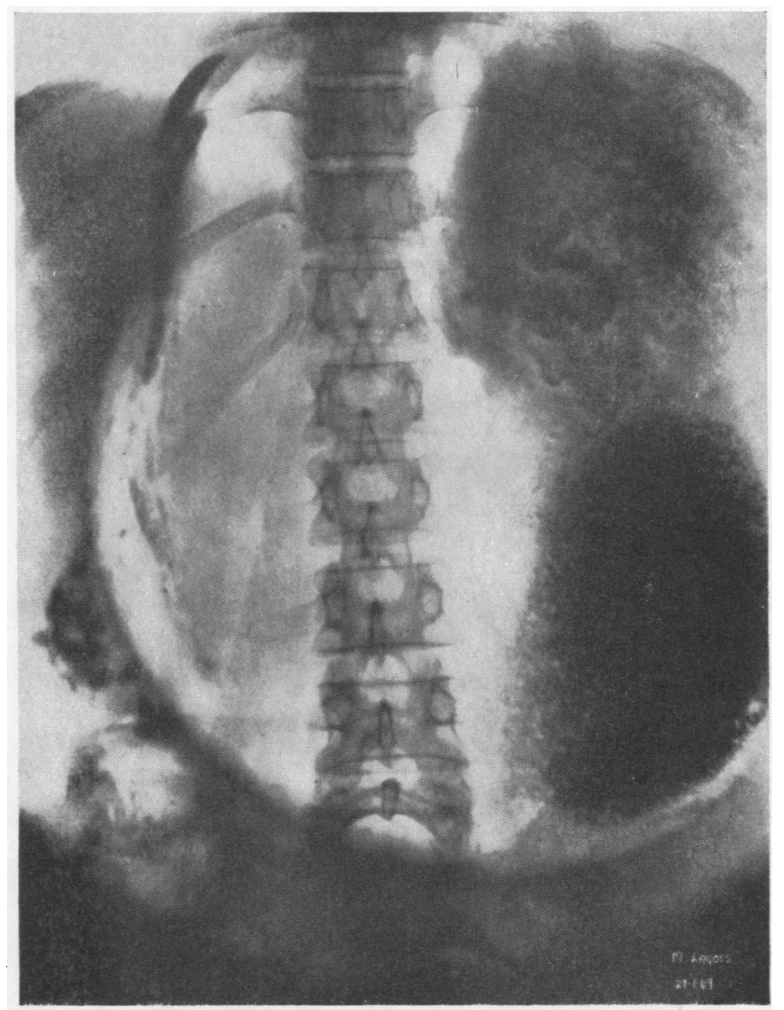

FIG. 4.-Antero-posterior radiograph (Case 7) of the abdomen taken in the supine position after ingestion of barium. It shows enormous gaseous distension of the body of the stomach due to obstruction of the pylorus in a hiatus hernia. A small quantity of barium has entered the duodenum opposite the medial part of the right diaphragm.

Diagnosis of hiatus hernia was possible in 15 cases on the characteristic appearances in the straight antero-posterior and lateral radiographs (Fig. 5). In the remaining four barium examination was necessary for diagnosis ; two had small herniae and two of the early patients had no lateral films taken. A barium meal and enema in the erect and Trendelenburg position were done as a routine. In all cases hernia contained stomach and stomach together with colon in three.

Operative reduction and repair was performed in 16 cases, and is always advised unless clearly contraindicated. In one, operation was withheld because of active pulmonary tuberculosis and two patients declined operation. Old people tolerate the operation well, and two patients aged 74 and 75 were successfully operated on. Sweet (1948) has emphasized the low mortality and excellent results of operative 


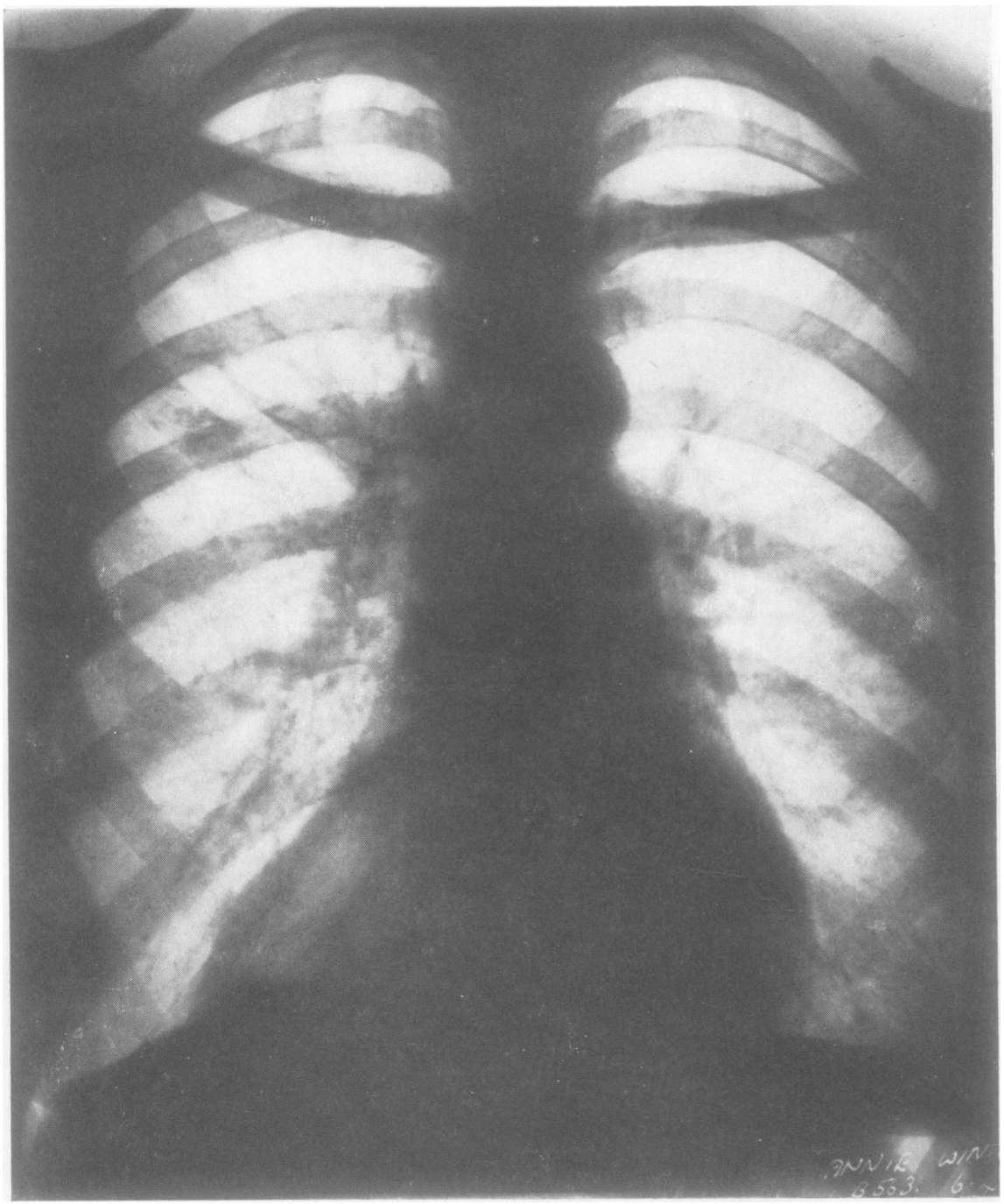

FiG. $5(a)$

FIG. 5.-(a) and (b) Antero-posterior and lateral radiographs showing the typical appearances of an oesophageal hiatus hernia. (c) Barium meal in the same case.

repair by the transthoracic route. Oesophagoscopy should be performed before operation in all cases. In our opinion severe oesophagitis, ulceration, stenosis or shortening of the oesophagus are contraindications to operation, as no relief of symptoms and recurrence of the hernia are likely.

The transthoracic route was employed in all cases. Operations on the diaphragm are undoubtedly made easier by controlled ventilation. We find anaesthesia with thiopentone, curare, nitrous oxide, and oxygen most suitable. Complete relaxation of the diaphragm is obtained and phrenic crush, which we consider undesirable, is 


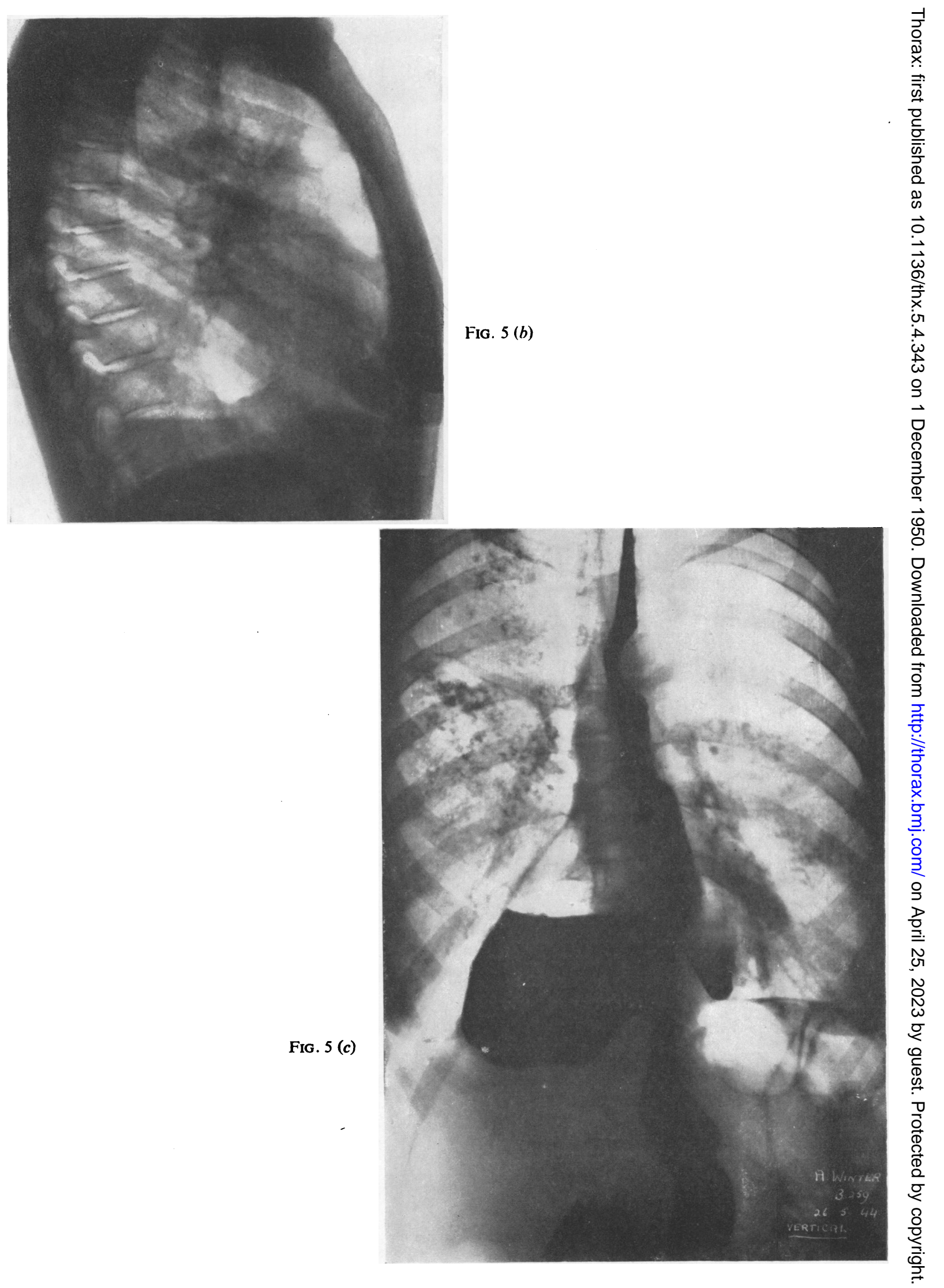


therefore unnecessary. In the early operations in the series the sac was opened, the contents reduced, and the hiatus narrowed from above. In the later cases the following transthoracic subdiaphragmatic approach developed by Mason (1947) was employed.

With the patient in the right lateral position, a left thoracotomy is performed through the bed of the eighth rib. The lung is retracted and the hernial sac identified. An incision planned to preserve the main branches of the phrenic nerve is made in the diaphragm (Fig. Y). The hiatus is then approached from the abdominal aspect

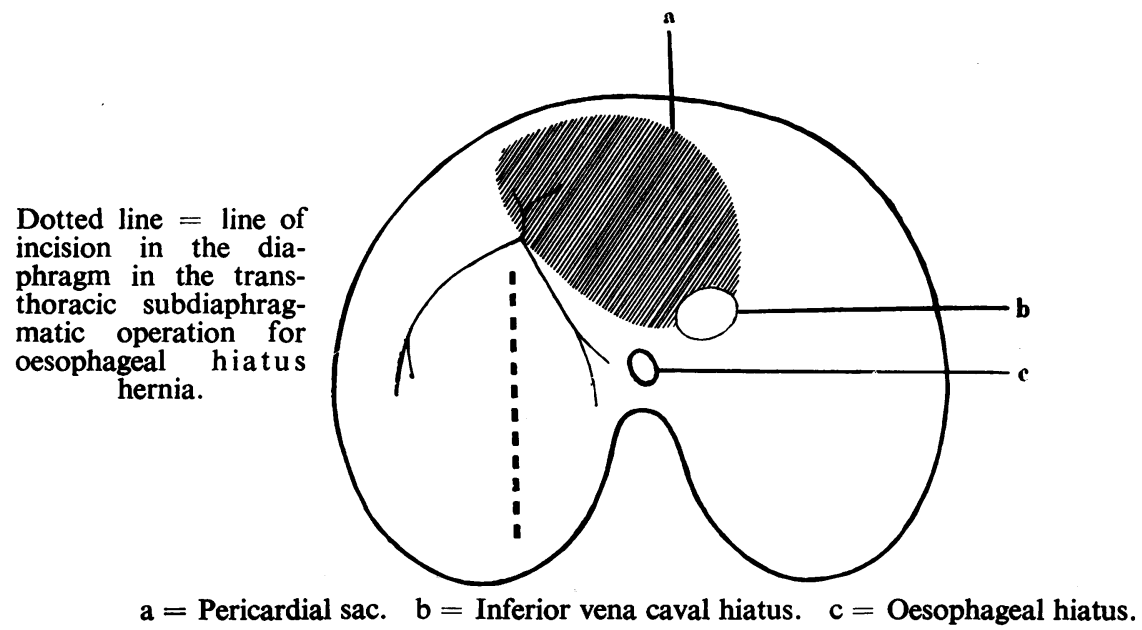

FIG. Y.-Diagram of diaphragm as seen from above, showing the branches of the left phrenic nerve.

and reduction and repair effected from below the diaphragm. The hernial contents are freed from the sac and a rubber catheter is passed as a sling around the oesophago-gastric junction. It is drawn gently downwards and forwards to bring the lower inch of the oesophagus below the diaphragm. The enlarged oesophageal hiatus is then visualized and interrupted sutures of No. 40 linen thread are inserted to approximate its margins behind the oesophagus. The oesophagus is anchored to the reconstituted hiatus with a few stitches. The incision in the diaphragm is closed with a double layer of continuous sutures. After re-expanding the lung the chest wall is closed in layers without drainage.

This technique was employed in 12 cases without recurrence of the herniation. Recurrence, however, occurred within six months in one of the five earlier cases in the series in which the repair had been performed entirely from above the diaphragm. In this case the repair was combined with a left lower lobectomy for bronchiectasis. At a second operation the hernia was repaired by the transthoracic subdiaphragmatic approach. The patient has been observed for two and a half years and there is no further recurrence.

There were no deaths after 17 operations on 16 cases. Post-operative management presented no special difficulty, and the patients were allowed up on the fifth day. Three developed collapse of the left lower lobe which re-expanded after bronchoscopy ; four developed effusions which required aspiration. All patients obtained complete symptomatic relief following operation except one, who still suffers from slight regurgitation on stooping, but her pain has been relieved. 
Hiatus Hernia with Oesophagitis. - Eight cases of hiatus hernia were associated with pathological changes in the oesophagus caused by regurgitation of acid gastric juice through an incompetent cardiac sphincter. Allison (1946) has described and classified the series of changes which occurs. Shortening of the oesophagus in these eight cases was demonstrated by biopsy showing gastric mucosa at distances varying between 32 and $36 \mathrm{~cm}$. from the upper incisor teeth in the adults and at $25 \mathrm{~cm}$. in the child (Case 8).

Barium examination in each case was necessary to demonstrate the hernia, which was small and not visible on straight radiographs. In all cases oesophageal symptoms were prominent. From our limited experience we consider that treatment should be directed at the oesophagus and not at the hernia. In two of our cases repair of the hernia was followed by return of symptoms with recurrence of herniation.

Two patients were seen in the early stage of acute oesophagitis and ulceration without stenosis. Both gave a history of flatulence, dyspepsia, and heartburn for many years and for one year they had complained of retrosternal pain on swallowing and regurgitation of food. In one of these, a woman aged 51, repair of the hiatus hernia was carried out; reduction was effected without undue tension ${ }^{\circ}$ on the oesophagus, and the oesophageal wall felt normal. Within six months symptoms had returned, though at this time no recurrence of the hernia could be seen ; three months later, however, a hernia was demonstrable. The other patient, a man aged 50 , responded to medical treatment-diet, alkalis, and elevation of the head of the bed at night-and has remained well.

Six were seen at a more advanced stage with stenosis of the lower end of the oesophagus. In one of these (Case 8), a child with symptoms dating from birth, reduction and repair of the hernia were attempted but there was recurrence soon afterwards.

Case 8 (No. 4173).-A girl of 13 had a history of frequent vomiting since birth and difficulty in swallowing solids. She was underweight and small for her age. Barium swallow showed narrowing of the lower end of the oesophagus associated with a small hiatus hernia. At oesophagoscopy a stricture of the lower end of the oesophagus was present. It was easily dilated and gastric mucosa was identified at $25 \mathrm{~cm}$. At operation there was a localized thickening and fibrosis of the lower end of the oesophagus, which was situated $1 \frac{1}{2}$ in. above the diaphragm. The hernia was reduced with difficulty, and suture of the lower end of the oesophagus to the margins of the reconstructed hiatus had to be done under tension. Vagotomy was performed. Radiographs two months later showed recurrence of the hernia. Little or no symptomatic relief was obtained and repeated dilatations have been required. Excision of the stricture is being considered.

Five cases were referred with a provisional diagnosis of carcinoma of the oesophagus. All were aged over 70 and gave a history of obstruction to swallowing for less than a year. At oesophagoscopy, varying degrees of oesophagitis and ulceration were seen and non-malignant strictures were present at distances varying from 32 to $36 \mathrm{~cm}$. from the upper incisor teeth. Dilatations at intervals of weeks or months, according to the severity of the symptoms, have been carried out and have enabled the patients to take an adequate diet.

Hernia Through Muscular and Tendinous Defects of the Diaphragm (10 Cases).- The cases in this group include one hernia through the foramen of Bochdalek (Case 9), one case of congenital absence of the posterior fourth of the 


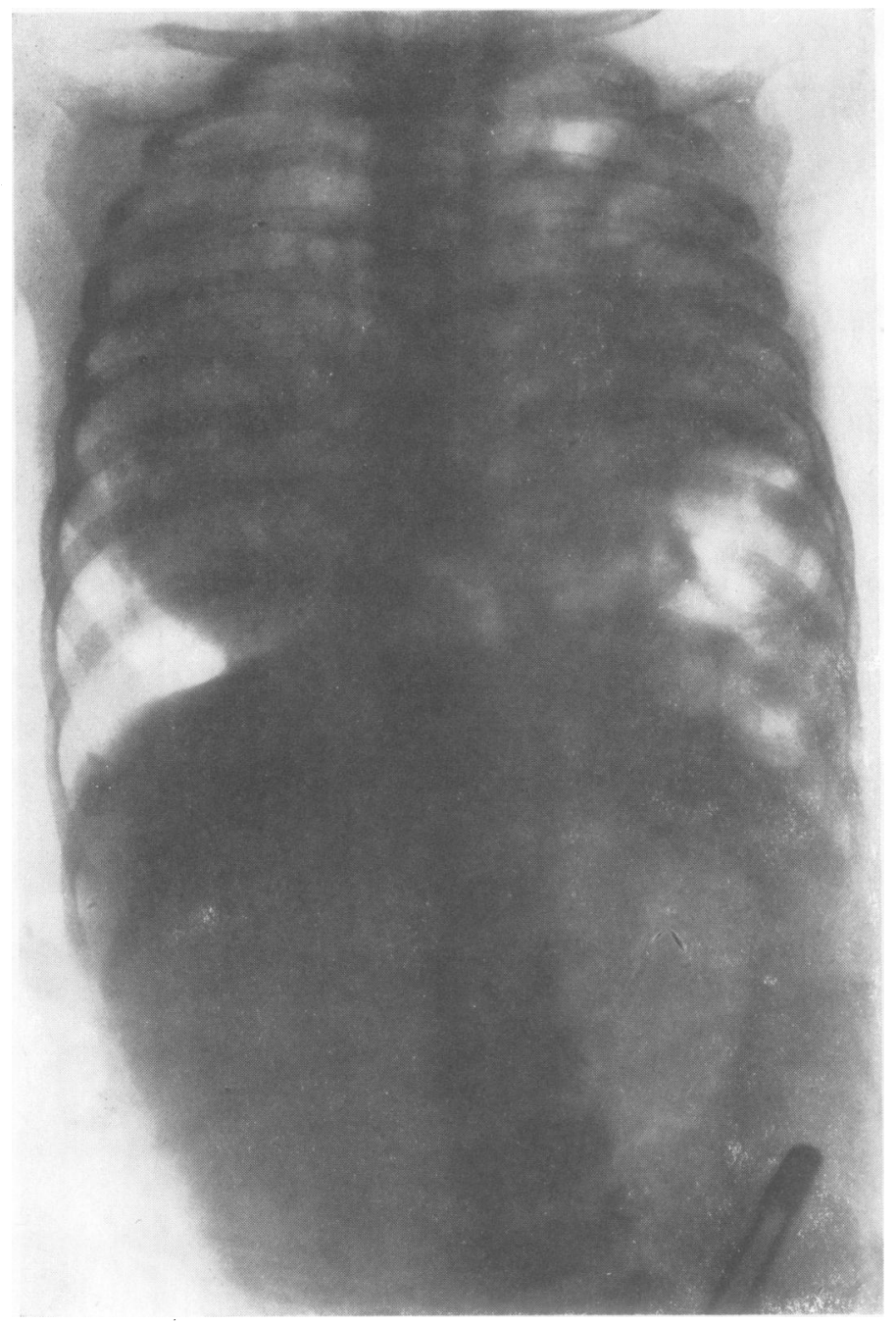

FIG. $6(a)$

FIG. $6(b)$

FIG. 6.-(a) Antero-posterior radiograph (Case 10) showing the left hemithorax occupied by coils of intestine with marked displacement of the heart to the right. (b) Radiograph showing barium in the small and large intestines.

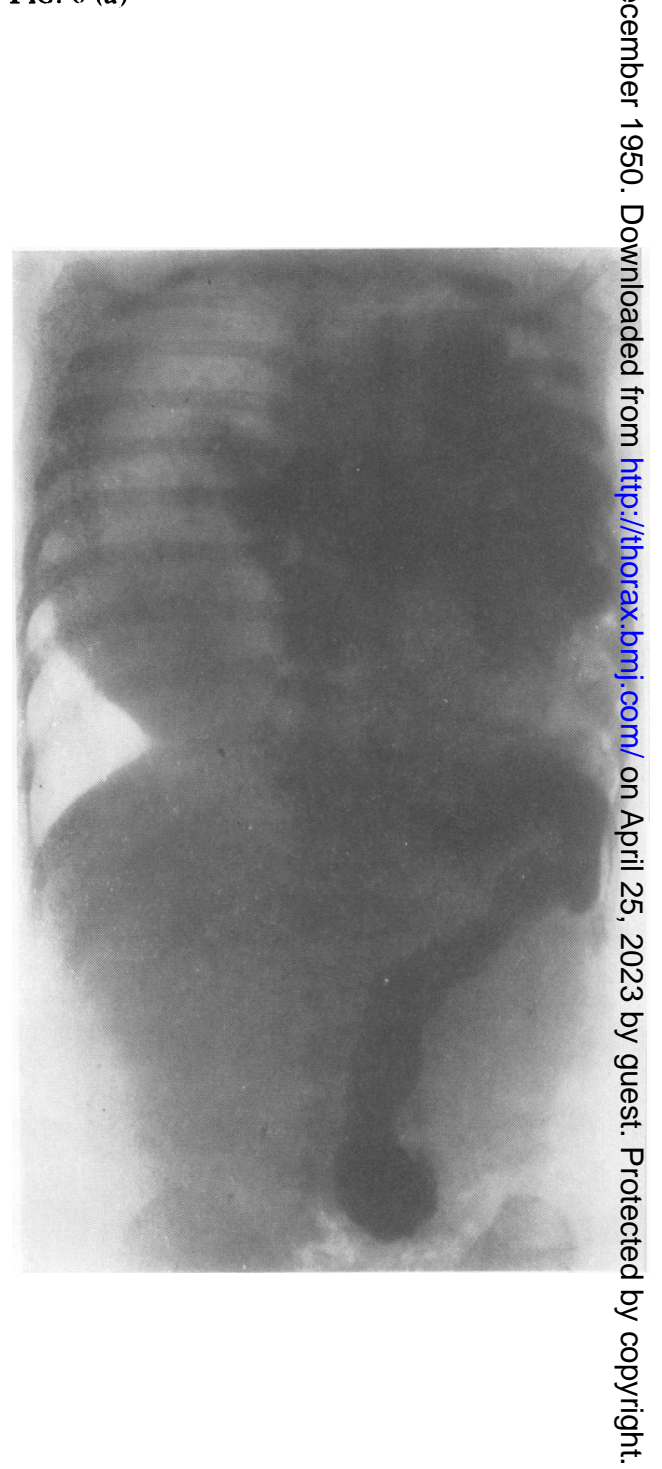


left hemidiaphragm (Case 10), one of hernia through the foramen of Morgagni, and seven of hernia through defects in various parts of the dome (Table I).

The severity of the symptoms is largely dependent on the size of the hernia, the size of the defect, and the presence or absence of a sac which may limit the herniation to some extent. Two infants (Cases 9 and 10) had very severe symptoms since birth and presented such difficult problems of management as were emphasized by Gross (1946). One of these (Case 10) was wasted and dehydrated on admission, and oral feeding was difficult because of choking attacks (Fig. 6). Operation was unfortunately deferred in the hope that the general condition could be improved, but bronchopneumonia supervened with a fatal outcome. At necropsy the left pleural cavity was occupied by coils of intestine and the left lung had never expanded. Early operation would have been advisable in such a case, and the more severe the symptoms the more urgent the indication. No improvement can be expected until the abdominal viscera have been replaced and the lung allowed to expand. In the other infant (Case 9) emergency operation was undertaken at special request in another hospital. After the administration of parenteral fluids, and under general anaesthesia, left thoracotomy was performed. The abdominal contents were replaced without difficulty and the defect in the diaphragm repaired in two layers. Following operation there was immediate improvement in the colour, which was now good without oxygen. The baby did well for two days, and radiographs showed full expansion of both lungs. During a feed on the third day she inhaled vomit and died suddenly. Necropsy revealed milk curds in the trachea with collapse of both lungs. Such a catastrophe should have been averted by avoidance of gastric distension and overfeeding.

In six cases the symptoms were mild or absent, and in four of these the hernia was discovered on routine radiological examination. In Case 14 differentiation from a cyst or tumour was not made pre-operatively (Fig. 7); the induction of a pneumoperitoneum might have clarified the diagnosis. In these six cases operative repair was performed by the thoracic route with no deaths or recurrences.

The following two cases presented as empyemata.

Case 16.-A woman aged 42 was admitted with a chronic left basal empyema, for which an unroofing operation was performed. Her vital capacity remained inexplicably low. Three months later when the empyema had almost healed she developed an attack of colicky upper abdominal pain and vomiting. Straight radiographs at this time were suggestive of diaphragmatic hernia, and the diagnosis was confirmed by barium examination. At operation a portion of the stomach and a knuckle of colon were found herniated through an opening 2 in. in diameter in the dome of the left diaphragm. The hernia was repaired and she eventually made a complete recovery, the vital capacity increasing from $700 \mathrm{ml}$. to $1,700 \mathrm{ml}$.

Case 17 (No. 2234). - A naval rating, aged 22, seven weeks before admission had developed sudden severe pain in the left chest with dyspnoea and pyrexia. He was thought to have pneumonia, and was given intramuscular penicillin. Three days later a pint of brownish fluid was aspirated from the left chest and penicillin instillations were begun. On admission to the thoracic unit he had a total left empyema with an unexpandable lung. Decortication of the lung was undertaken, and during the operation fluid containing undigested food was seen to well up into the lower part of the paravertebral gutter. On further exploration a portion of the stomach with a large hole in its wall was encountered. The stomach had herniated through a defect, $1 \mathrm{in}$. in diameter, in the posterior part of the dome of the left diaphragm. The stomach was sutured and 
TABLE 1

Herniae through the Muscular and Tendinous Portions of the Diaphragm

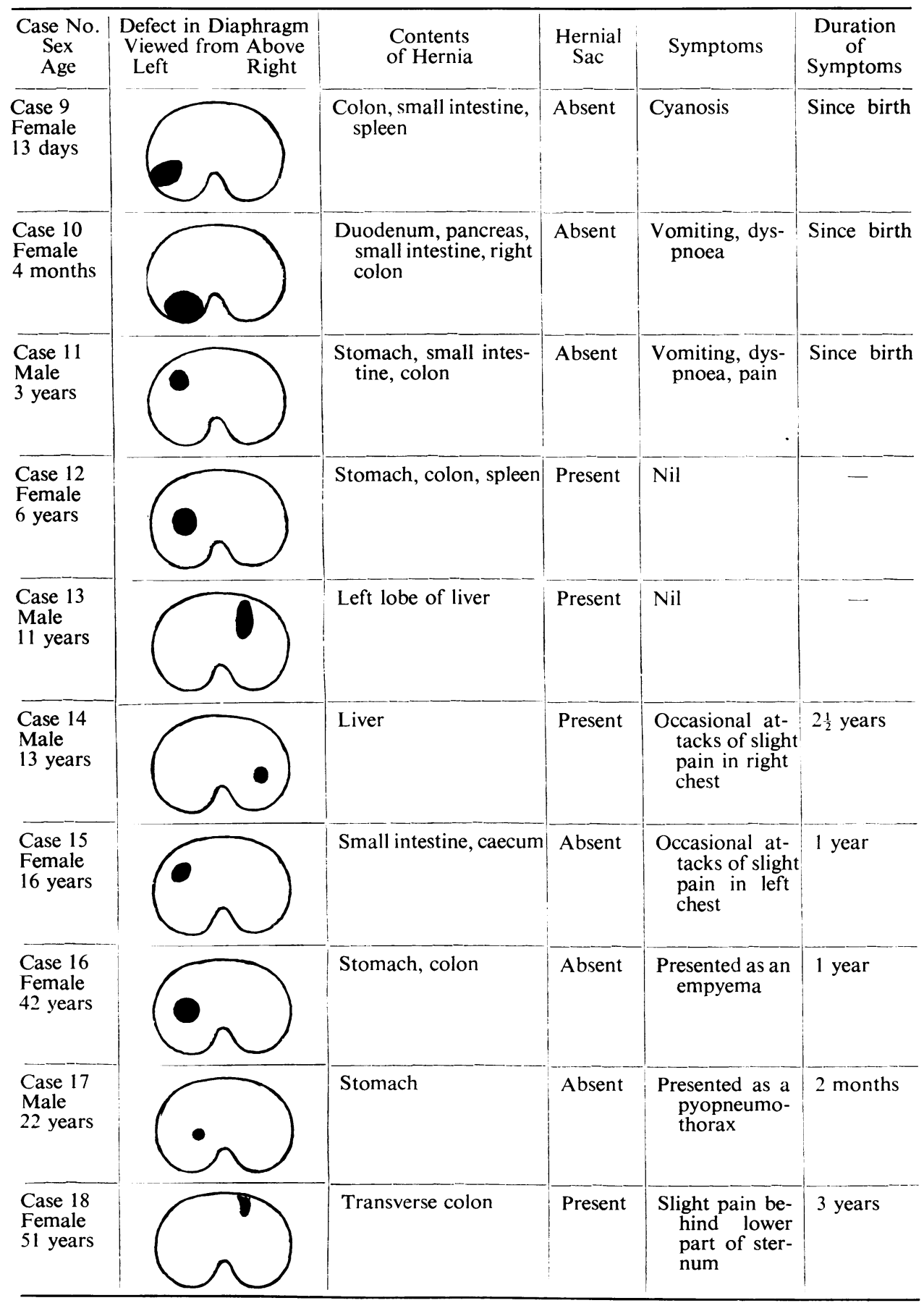




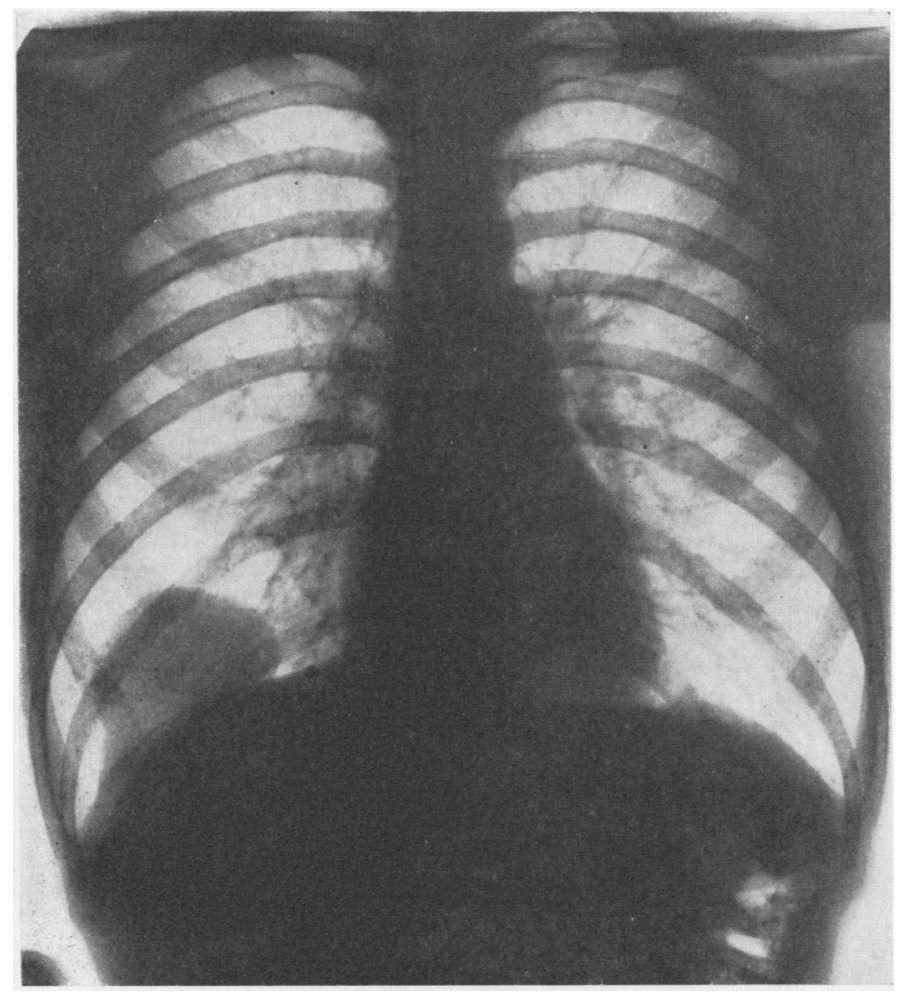

FIG. $7(a)$

FIG. $7(b)$

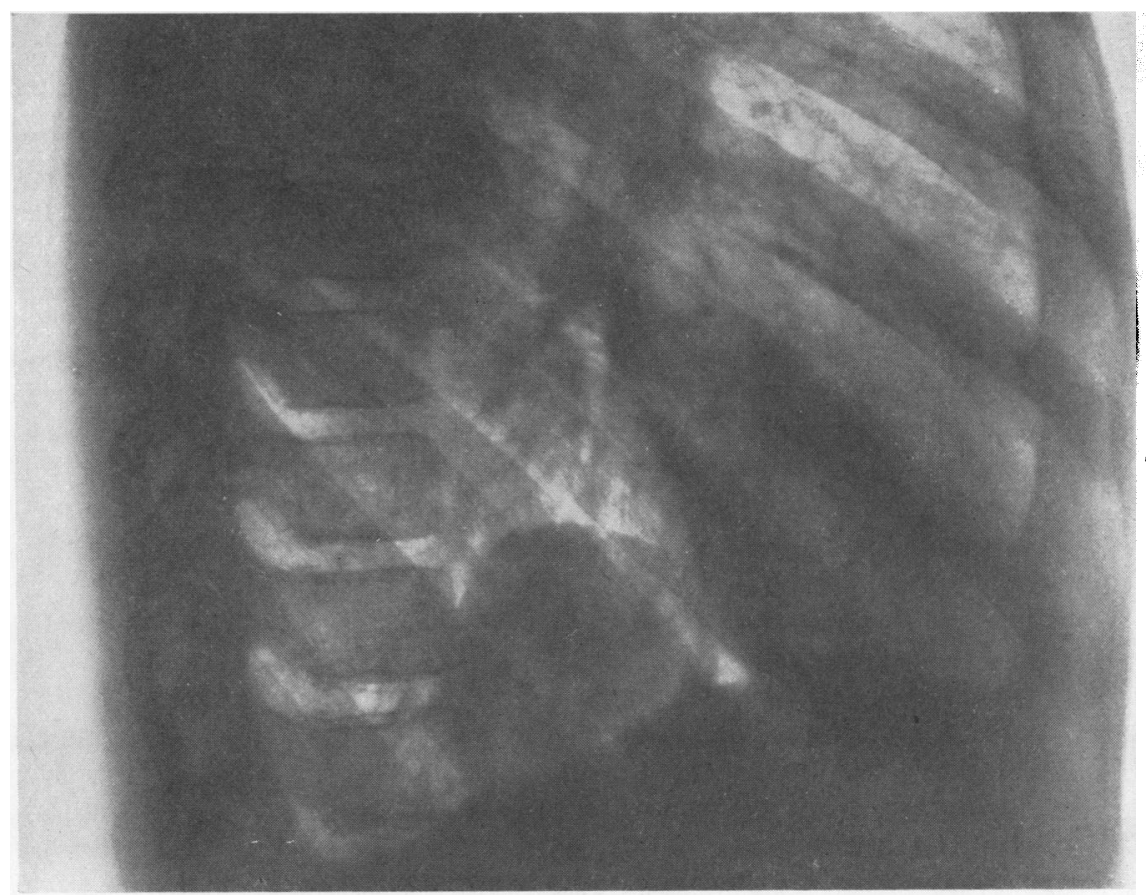

FIG.7 (a) and (b) - - Antero-posterior and right lateral radiographs (Case 14) of the chest showing herniation of a portion of the liver through a defect in the dome of the right diaphragm. 
the decortication completed. Following the operation his lung re-expanded and the empyema healed. Five months later the hernia was repaired by the thoracic route. No evidence of gastric ulcer was to be found and he made a good recovery. It is difficult to explain the pathology in this case, but it is possible that strangulation of the herniated stomach had occurred with necrosis and sloughing of a portion of its wall.

\section{Eventration of THE Diaphragm}

Differentiation between this condition and diaphragmatic hernia may be difficult and therefore it is convenient to review them together.

Eight cases of eventration have been admitted to this unit since 1937. Seven were men and one was a woman; their ages varied from 2 to 58 years.

In all the left diaphragm was affected. In two the dome of the diaphragm reached the level of the sixth dorsal vertebra and in the others it was elevated to a lesser degree. Diminished excursion in the normal direction was present in six and in the other two the affected diaphragm was immobile.

Barium examination showed inversion of the stomach in four cases, giving the characteristic inverted fishhook appearance described by Rosenfeld (1944). The stomach rotated so that the greater curvature was uppermost under the elevated dome of the diaphragm ; the pylorus and pyloric antrum lay anteriorly in the left upper quadrant of the abdomen (Fig. 8).

A barium enema was given in five cases and showed the splenic flexure to be immediately under the elevated diaphragm. In a boy of 2 years who had had symptoms since birth, the whole of the transverse colon was in close contact with the diaphragm, which was elevated to the level of the aortic arch, and the caecum was situated in the epigastrium.

All, except one patient, had symptoms, but in no instance were these severe or incapacitating. In six cases the symptoms were referable to the chest and included dyspnoea on exertion, cough, and attacks of pain in the left side. Two children had a history of repeated febrile attacks with cough and expectoration. Abdominal symptoms were less common; attacks of vomiting had occurred in three cases, of which only one had inversion of the stomach.

Reed and Borden (1935) consider that the diagnosis of eventration rests on the radiological visualization of an intact diaphragm as an unbroken line. This was made more obvious in one of our cases by the induction of a pneumoperitoneum (Fig. 9). Movement of the elevated diaphragm in the normal direction favours a diagnosis of eventration rather than hernia. This movement may be very slight and may even be regarded as paradoxical unless each leaf of the diaphragm is observed separately. Differentiation is not always possible: even at operation it is sometimes difficult to distinguish between a localized eventration and a diaphragmatic hernia with a wide neck and a complete thick-walled sac.

There are very few references in the literature to the surgical treatment of eventration. Lerche (1927) describes one case of eventration which was plicated by the abdominal route. Bisgard (1947) reports a case in which plication was performed by the thoracic route and could only find records of five previous cases. One died three days post-operatively, but the remaining five cases were improved by operation.

Operation was performed in four of our cases. In three of these differentiation between hernia and eventration was not made before thoracotomy. The elevated 


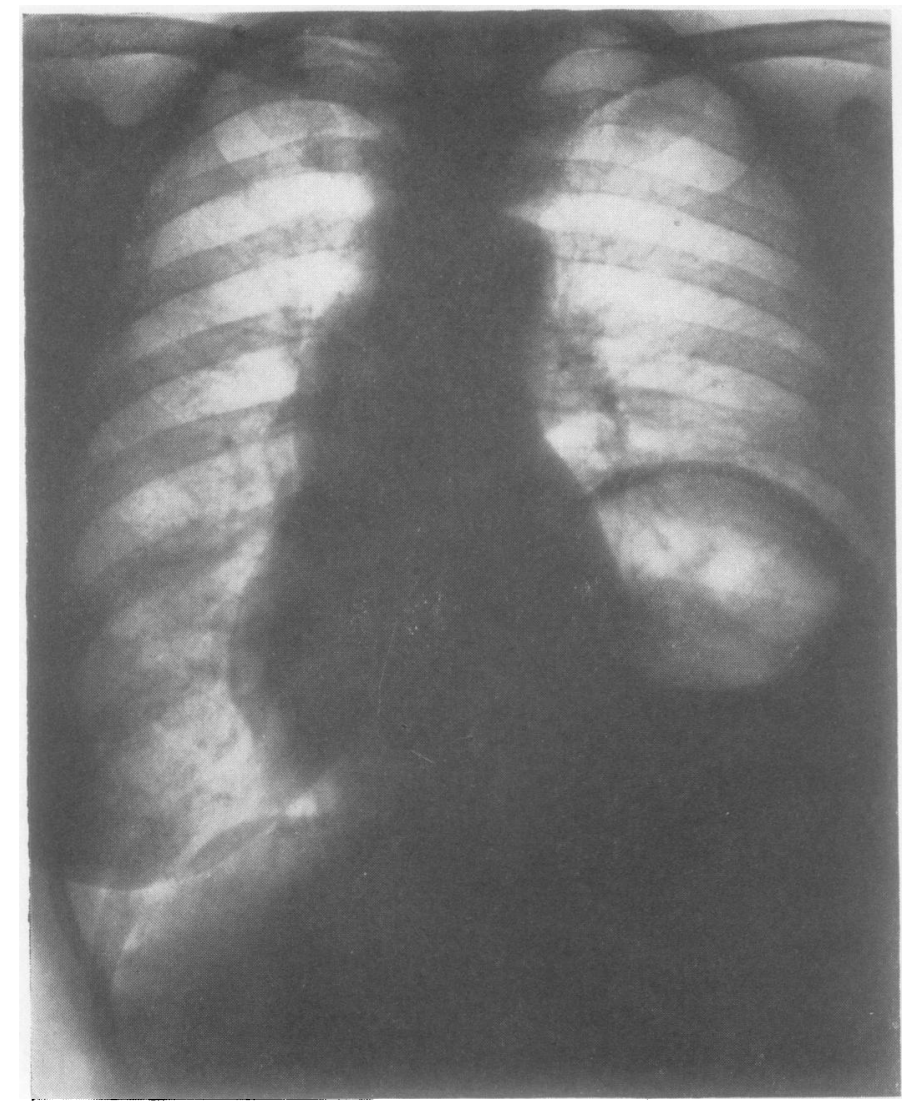

FIG. $9(a)$

FIG. $9(b)$

FIG. 9.-(a) Antero-posterior radiograph showing eventration of the left diaphragm. (b) Anteroposterior radiograph in the same case after induction of a pneumoperitoneum and showing the unbroken line of the left diaphragm.

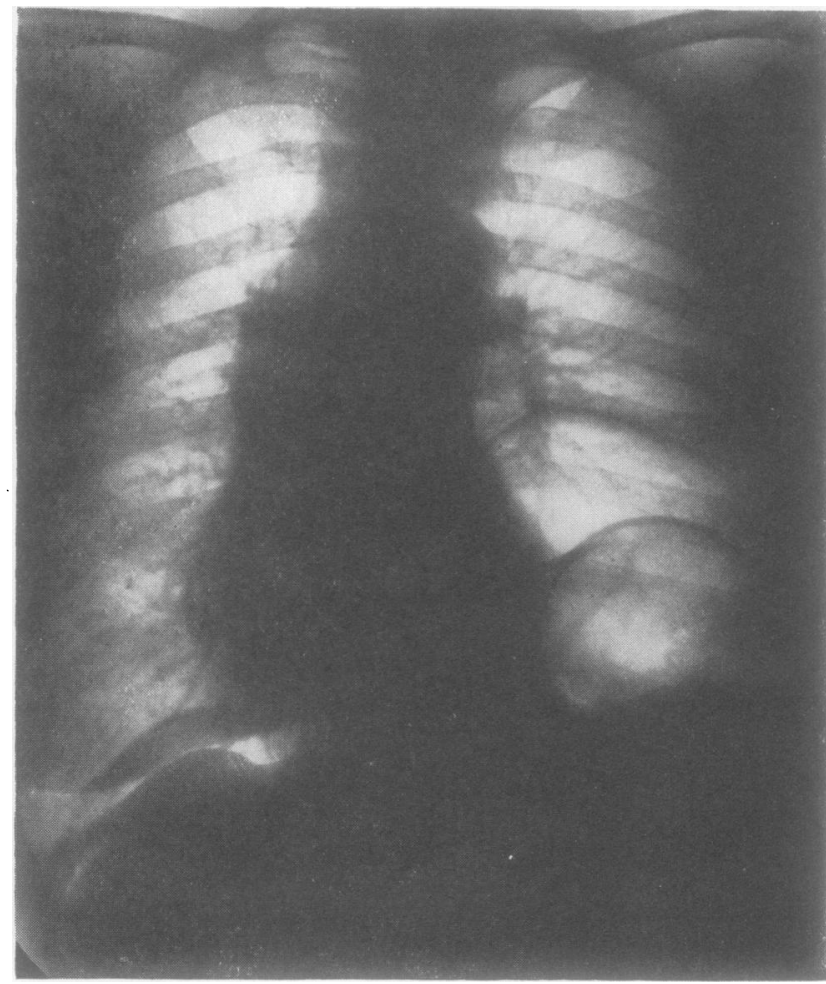


diaphragm was thinner than normal but held sutures without tearing. In two, only the anterior two-thirds appeared to be attenuated; in the other two the whole of the diaphragm was uniformly involved. The phrenic nerve was always of normal appearance and calibre. In three cases the diaphragm was plicated and in one incised, overlapped, and sutured.

Sufficient time has not elapsed since these operations to make a final assessment. In three cases symptoms have been relieved (the fourth was symptomless preoperatively). There is in each case an appreciable lowering of the level of the affected diaphragm ; in three the diaphragm is immobile and the fourth shows slight paradoxical movement. Inversion of the stomach, which had been present in three cases, has been corrected.

\section{SUMMARY}

Forty-nine cases of diaphragmatic hernia are classified and reviewed. No differentiation into congenital and acquired types is made.

The wide variation in the severity of the causative injury and in the symptomatology of the traumatic cases is illustrated. The association between traumatic hernia and fractured pelvis is recorded.

Certain complications of herniation which demand urgent operation are illustrated, including a strangulated hernia following cardio-omentopexy.

Transthoracic operation is advised, and the results of 40 operations are given. A transthoracic subdiaphragmatic repair for oesophageal hiatus herniae is described and was performed in 12 cases with no deaths or recurrences.

Operative repair of oesophageal hiatus hernia is not recommended in the presence of pathological changes in the oesophagus.

Eight cases of eventration of the left diaphragm, including four upon which operation was performed, are presented.

Our thanks are due to Mr. George A. Mason for his valuable help and encouragement in the preparation of this paper; to Dr. Whately Davidson for his co-operation in the investigation of the cases and in the interpretation of the radiographs; to $\mathrm{Mr}$. W. Buckley for the records of the three cases from the Nottingham General Hospital; to Dr. E. Joan Millar for her advice on anaesthesia; and to Professor Green and Professor Short, of the Department of Anatomy, King's College Medical School, Newcastle-upon-Tyne, for facilities for dissecting specimens.

\section{REFERENCES}

Allison, P. R. (1946). J. thorac. Surg., 15, 308.

Barrett, N. R., and Wheaton, G. E. W. (1934). Brit. J. Surg., 21, 420.

Bisgard, J. D. (1947). J. thorac. Surg., 16, 484.

Donovan, E. J. (1945). Ann. Surg., 122, 569.

Dugan, D. J., and Samson, P. C. (1948). J. thorac. Surg., 17, 771.

Dunhill, T. (1935). Brit. J. Surg., 22, 475.

Gross, R. E. (1946). Amer. J. Dis Child., 71, 579.

Harrington, S. W. (1940). Amer. J. Surg., 50, 377.

- (1945). Ann. Surg., 122, 546.

Hughes, F., Kay, E. B., Meade, R. H., Hudson, T. R., and Johnson, J. (1948). J. thorac. Surg., $17,99$.

Lerche, W. (1927). Arch. Surg., Chicago, 14, 285.

Mason, G. A. (1947). Personal communication.

Morton, J. J. (1939). Surg. Gynec. Obstet., 68, 257.

Olsen, A. M., and Harrington, S. W. (1948). J. thorac. Surg., 17, 189.

Orr, I. M. (1946). Brit. J. Surg., 34, 97.

Reed, J. A., and Borden, D. L. (1935), Arch. Surg., Chicago, 31, 30.

Rosenfeld, D. H. (1944). Amer. J. Roentgenol., 52, 607.

Sweet, R. H. (1948). New Engl. J. Med., 238, 649.

Truesdale, P. E. (1945). J. thorac. Surg., 14, 160. 